\section{POS0121 RESPONSE OF EOSINOPHILIC GRANULOMATOSIS WITH POLYANGIITIS TO MEPOLIZUMAB ACCORDING TO DISEASE MANIFESTATIONS. A SINGLE CENTRE EXPERIENCE}

R. Ríos-Garcés ${ }^{1}$, S. Prieto-González ${ }^{1}$, J. Hernández-Rodríguez ${ }^{1}$, M. C. Cid ${ }^{1}$, G. Espígol-Frigolé1. ${ }^{1}$ Hospital Clínic de Barcelona, Systemic Autoimmune Diseases, Barcelona, Spain

Background: Eosinophilic granulomatosis with polyangiitis (EGPA) is a relapsing disease with frequent glucocorticoid (GC) dependence. Mepolizumab (MEPO) has been demonstrated to reduce flares and spare GC. However, EGPA is a heterogeneous disease and the effects of MEPO on specific disease manifestations has not been completely delimitated.

Objectives: To analyze the impact of MEPO on manifestations derived from small-vessel vasculitis, ENT symptoms, asthma, eosinophilic tissue infiltration and ANCA status in a single-centre cohort of EGPA patients.

Methods: Medical chart of EGPA patients treated with MEPO were reviewed to describe demographics, clinical characteristics, steroid dose at the initiation of MEPO and during follow-up, flares, disease activity, damage accrual and laboratory results.

Results: Among 52 EGPA patients regularly controlled in our department, 11 patients were treated with MEPO. MEPO was prescribed when a) patients required prednisone (PDN) at $\geq 7.5 \mathrm{mg} / \mathrm{d}$ to maintain stability, or b) when maintained with $<7.5 \mathrm{mg} / \mathrm{d}$, presented at least 4 exacerbations/year requiring an increase in PDN dose. 6 were males and 5 females, with a mean age of 54 years at MEPO initiation. Baseline characteristics of the patients and course under treatment are presented in Table 1. ENT involvement, followed by asthma and eosinophil-related tissue-infiltration (ETI) were the most common symptoms when prescribing MEPO. Regarding treatment, patients received MEPO at 100$300 \mathrm{mg} \mathrm{SC}$ monthly. The definition of flare was the same used in the MIRRA trial ${ }^{1}$. The mean time of treatment with MEPO was 34 months. All patients achieved a BVAS score of 0 points at 12 months or earlier. In general, patients reduced the number of flares, which tended to be milder, and all related to asthma or ENT manifestations. All improved their asthma control, but 3 of them persisted with recurrent ENT symptoms in spite of treatment with MEPO. None of them had vasculitic manifestations (cutaneous, neurological, gastrointestinal, renal) manifestations during treatment. All patients were able to tapper their PDN dose to $\leq 5 \mathrm{mg} /$ day or less, except 3 patients. Of the 3 patients who required $\geq 5 \mathrm{mg} / \mathrm{d}$, 1 had severe asthma, but diminished the previous PDN dose $(22.5 \mathrm{mg} / \mathrm{d}$ preMEPO, $10 \mathrm{mg} / \mathrm{d}$ currently) and the yearly rate of flares (8.2 pre-MEPO, 0.64 under-MEPO). The other one notably improved his asthma, but had ENT symptoms that responded unsatisfactorily to MEPO and required a maintenance PDN dose of $7.5 \mathrm{mg} / \mathrm{d}$. The last one, improved her asthma control and was able to begin PDN tapering, but persisted with ENT symptoms. Regarding damage accrual, 6 patients remained stable during treatment, and 5 worsened. Two of three ANCA positive patients remained positive in spite of treatment.

Table 1.

\begin{tabular}{|c|c|c|c|}
\hline & $\begin{array}{l}\text { Baseline characteris } \\
\text { tics at diagnosis }\end{array}$ & $\begin{array}{l}\text {-At mepolizumab } \\
\text { initiation }\end{array}$ & $\begin{array}{l}\text { After mepoli- } \\
\text { zumab (last } \\
\text { follow-up) }\end{array}$ \\
\hline Age, median (range) years & $49(23-67)$ & $54(35-69)$ & - \\
\hline Male/Female, $\mathrm{n}(\%)$ & $6 / 5(54.5 \% / 45.5 \%)$ & - & - \\
\hline BVAS, median (range) & $11(2-20)$ & $2(0-6)$ & 0 \\
\hline FFS, value (n, \%) & $1(1,9.1 \%)$ & - & - \\
\hline VDI, mean (range) & - & $1.7(0-5)$ & $2.3(0-5)$ \\
\hline Asthma, n (\%) & $11(100 \%)$ & $3(27.3 \%)$ & $0(0 \%)$ \\
\hline ENT, n (\%) & $10(90 \%)$ & $4(36.4 \%)$ & $3(27.3 \%)$ \\
\hline SVV, n (\%) & $4(36.4 \%)$ & $0(0 \%)$ & $0(0 \%)$ \\
\hline ETI signs/symptoms, n (\%) & $7(63.6 \%)$ & $2(18.2 \%)$ & $0(0 \%)$ \\
\hline Constitutional symptoms, n (\%) & $4(36.4 \%)$ & $0(0 \%)$ & $0(0 \%)$ \\
\hline $\begin{array}{l}\text { Eosinophils, mean (range) cells } \\
\times 10^{9}\end{array}$ & $5500(600-8850)$ & $240(0-600)$ & $55(0-200)$ \\
\hline ANCA positivity (IIF) & $7(77.8 \%)$ & 3 & 2 \\
\hline Anti-MPO titers, mean (range) & $286(93-740)$ & $88(3-739)$ & $7(3-37)$ \\
\hline Yearly rate of flares & - & 1,75 & 0.51 \\
\hline Immunosuppressants, $\mathrm{n}$ & - & 3 & 1 \\
\hline Prednisone dose, mg/d (range) & $8(7.5-25)$ & $11.4(5-22.5)$ & $5.125(0-10)$ \\
\hline
\end{tabular}

Conclusion: MEPO was effective for the treatment of patients with EGPA, with a reduction in the number and severity of flares and a decrease in PDN doses. A worse response of ENT involvement was observed. No vasculitic flares were observed in spite of GC reduction. Mepolizumab did not prevent damage accrual during the treatment period.

\section{REFERENCES:}

[1] Wechsler ME et al. MEPO or Placebo for Eosinophilic Granulomatosis with Polyangiitis. N Engl J Med. 2017.
Acknowledgements: Funding: Plan Estatal de Investigación Científica y Técnica y de Innovación 2013-2016 (PI18/00461), co-funded by Instituto de Salud Carlos III (ISCIII) and Fondo Europeo de Desarrollo Regional (FEDER) and by Río Hortega program (ISCIII, CM19/00032).

Disclosure of Interests: Roberto Ríos-Garcés: None declared, Sergio Prieto-González: None declared, José Hernández-Rodríguez: None declared, Maria C. Cid Paid instructor for: GSK and Vifor, Consultant of: GSK, Abbvie and Janssen, Grant/research support from: Kiniksa and Roche, Georgina Espígol-Frigolé Consultant of: Janssen, Grant/research support from: Roche DOI: 10.1136/annrheumdis-2021-eular.2852

\section{Central pain and other extra-articular associations to KOA}

\begin{tabular}{|l|l|}
\hline POS0122 & THE ASSOCIATIONS BETWEEN DISEASE MODIFYING \\
ANTI-RHEUMATIC DRUGS AND INCIDENT, AND \\
PROGRESSION OF, RADIOGRAPHIC HAND \\
OSTEOARTHRITIS IN RHEUMATOID ARTHRITIS \\
PATIENTS
\end{tabular}

T. Burkard ${ }^{1}$, C. Lechtenboehmer ${ }^{2}$, S. Reichenbach ${ }^{3}$, M. Hebeisen ${ }^{4}$, U. Walker ${ }^{5}$, A. M. Burden ${ }^{1,6}$, T. Hügle ${ }^{7}{ }^{1}{ }^{1}$ TTH Zurich, Department of Chemistry and Applied Biosciences, Zurich, Switzerland; ${ }^{2}$ University Hospital of Basel, Department of Radiology, Basel, Switzerland; ${ }^{3}$ University of Bern, Institute for Social and Preventive Medicine, Bern, Switzerland; ${ }^{4}$ SCQM Foundation, SCQM Foundation, Zurich, Switzerland; ${ }^{5}$ University Hospital of Basel, Department of Rheumatology, Basel, Switzerland; ${ }^{6}$ Maastricht University Medical Center+, Department of Clinical Pharmacy and Toxicology, Maastricht, Switzerland; ${ }^{7}$ Lausanne University Hospital (CHUV) and University Lausanne, Department of Rheumatology, Lausanne, Switzerland

Background: Hand osteoarthritis (OA) is characterized by bone erosions, joint space remodeling, and new bone formation mainly in distal interphalangeal (DIP) joints and thereby differs from hand manifestations in rheumatoid arthritis (RA). There are conflicting data about the benefit of treatment with conventional synthetic (cs) and biologic (b) disease modifying anti-rheumatic treatment (DMARD) on DIP OA.

Objectives: To assess the associations between DMARDs and incident, and progression of, radiographic DIP OA in RA patients.

Methods: We performed two observational cohort studies in the longitudina Swiss Clinical Quality Management in Rheumatic Diseases registry (SCQM) between 1997 and 2014. RA patients who had $\geq 2$ eligible hand radiographs were included at their first eligible radiograph (baseline) and were followed until the outcome or their last eligible radiograph. Radiographs were eligible if all 8 DIP joints could be scored. Modified Kellgren-Lawrence scores (KLS) were obtained by evaluating DIP joints for severity of osteophytes, joint space narrowing, subchondral sclerosis, and erosions. Incident/existing DIP OA was defined as KLS $\geq 2$ in $\geq 1$ DIP joint. Progression of existing DIP OA was defined as an increase of $\geq 1$ in KLS in $\geq 1$ DIP joint. We divided the study population into two cohorts based on whether DIP OA was present or absent at cohort entry (cohorts 1 and 2, respectively). Exposure status was defined time-dependently into mutually exclusive exposure groups: csDMARD monotherapy, bDMARD monotherapy, bDMARD/csDMARD combination therapy, past bDMARD/csDMARD therapy, or never DMARD use. Cox time-varying proportional hazard regression analyses were used to estimate hazard ratios (HRs) with $95 \%$ confidence intervals $(\mathrm{Cl})$ of DIP OA progression (cohort 1) or DIP OA incidence (cohort 2) associated with DMARD exposure categories (csDMARD monotherapy was the reference group because it was the largest group). Exposure and covariate information were extracted at every radiograph and other visit date. Missing covariate information was imputed using multiple imputation by chained equations. In sensitivity analyses, we repeated all analyses using generalised estimation equations (GEE). Results: Among 2234 RA patients with 5928 eligible radiographs, 1340 patients had radiographic DIP OA at cohort entry (cohort 1) and 894 were DIP OA naïve (cohort 2). In cohort 1, radiographic progression of existing DIP OA was characterized by new osteophyte formation $(666,52.4 \%)$, followed by joint space narrowing $(379,27.5 \%)$, subchondral sclerosis $(238,17.8 \%)$, and erosion (62, $4.3 \%$ ). bDMARD monotherapy was associated with an increased risk of radiographic DIP OA progression compared to csDMARD monotherapy (adjusted HR $1.34,95 \% \mathrm{Cl} 1.07-1.69)$. The risk of DIP OA progression was not significant in csDMARD/bDMARD combination therapy users (adjusted HR 1.12, 95\% Cl 0.96-1.31), absent in past DMARD users (adjusted HR 0.96, 95\% Cl 0.66-1.41), and significantly lower among never DMARD users (adjusted HR $0.54,95 \% \mathrm{Cl}$ 0.33-0.90), compared to csDMARD monotherapy users. In cohort 2, the risk of incident $\mathrm{OA}$ did not differ materially between treatment groups. Results from GEE analyses corroborated all findings. 